\title{
CONTRIBUTIONS TO PROJECTIVE THEORY OF SINGULAR POINTS OF SPACE CURVES
}

\author{
BY \\ SU-CHENG CHANG
}

In a recent paper $\left.{ }^{1}\right)$ we have obtained some covariant figures associated with a point of inflexion of a space curve and arrived at the semi-canonical expansions. But soon Professor $\mathrm{Su}\left({ }^{2}\right)$ improved the theory by establishing canonical expansions of a space curve at its point of inflexion $P$ in virtue of the neighborhood at $P$ of order nine. Naturally we may ask whether or not nine is the least order of the neighborhood of which we can geometrically define a covariant tetrahedron of reference and the unit point. The answer is negative. The object of this paper is to demonstrate that the eighth order neighborhood of the curve at its inflexion suffices for this purpose.

In both Su's paper and ours the projective differential geometry of singular points of plane curves has played an important role, whereas we shall develop here a new theory by an elementary and direct method.

1. Let $(x, y, z)$ be nonhomogeneous coordinates of a point in space, then upon selecting the point of inflexion $P$ of the curve for $(0,0,0)$, we can write the expansions of the curve at $P$ in the form

$$
\begin{aligned}
& y=A x^{3}+B x^{4}+C x^{5}+D x^{6}+E x^{7}+F x^{8}+(9), \\
& z=R x^{4}+S x^{5}+T x^{6}+U x^{7}+V x^{8}+(9),
\end{aligned}
$$

where $y=0, z=0$ denote the tangent at $P$ and $z=0$ the hyperosculating plane of the curve (1).

A quadric which has at $P$ a contact of order four with the curve (1) is found to be

$$
\frac{R}{A} x y-z+\alpha_{7} y^{2}+\alpha_{8} x z+\alpha_{9} y z+\alpha_{10} z^{2}=0,
$$

$\alpha_{7}, \alpha_{8}, \alpha_{9}$, and $\alpha_{01}$ being arbitrary constants. In order that the above quadric should have at $P$ a contact of order five with (1), it is necessary and sufficient that

$$
\frac{R}{A} B-S+\alpha_{8} R=0
$$

Presented to the Society, February 22, 1947; received by the editors December 17, 1945.

(1) S. C. Chang, On the point of inflexion of a space curve, Revista, Universidad Nacional de Tucumán, Argentina, serie A.

(2) B. Su, A note on the singularity of a space curve, Revista, Universidad Nacional de Tucumán, Argentina, serie A. 
so that (2) becomes

$$
\frac{R}{A} x y-z+\alpha_{7} y^{2}+\frac{1}{R}\left(S-\frac{R B}{A}\right) x z+\alpha_{\theta} y z+\alpha_{10} z^{2}=0 .
$$

At every point $Q\left(t_{0}, x_{0}, 0,0\right)$ on the singular tangent, the tangent plane to all the quadrics (4) coincide with each other, namely,

$$
\frac{R}{A} x_{0} y-t_{0} z+\frac{1}{R}\left(S-\frac{R B}{A}\right) x_{0} z=0,
$$

which defines a projectivity $P_{5}$ between $Q$ and a plane through the singular tangent.

For the sake of convenience we take the corresponding plane under $P_{5}$ of $(0,1,0,0)$ as $y=0$. This implies that

$$
A S-B R=0,
$$

and therefore that (4) reduces to

$$
\frac{R}{A} x y-z+\alpha_{7} y^{2}+\alpha_{9} y z+\alpha_{10} z^{2}=0 .
$$

The order of contact at $P$ between the curve (1) and the quadrics (7) becomes six if and only if

$$
\alpha_{7}=\frac{T}{A^{2}}-\frac{R C}{A^{3}} .
$$

Consequently, besides $y=0, z=0$ all the quadrics (7) have another common generator:

$$
z=0, \quad \frac{R}{A} x+\left(\frac{T}{A^{2}}-\frac{R C}{A^{3}}\right) z=0 .
$$

Taking the latter for $x=0, z=0$ gives that

$$
A T-R C=0 .
$$

When and only when

$$
\frac{R}{A} D-U+\alpha_{9} A R=0,
$$

all the quadrics

$$
\frac{R}{A} x y-z+\alpha_{9} y z+\alpha_{10} z^{2}=0
$$

have at $P$ a contact of order seven with the curve (1). In this case the tangent 
planes of these quadrics at

$$
\left(t_{0}, 0, y_{0}, 0\right)
$$

become one and the same plane

$$
\frac{R}{A} y_{0} x-t_{0} z+\alpha_{9} y_{0} z=0 .
$$

Thus we have reached a projectivity $P_{7}$ between the points on the covariant line (9) and planes turning around it. It is natural to take the corresponding plane of $(0,0,1,0)$ for $x=0$, so that $\alpha_{9}=0$, that is

$$
R D-A U=0 .
$$

In the pencil (12). the only quadric which has at $P$ a contact of order eight with (1) is given by the equation

$$
\frac{R}{A} x y-z+\frac{1}{R^{2}}\left(V-\frac{R E}{A}\right) z^{2}=0 .
$$

If we take $(0,0,0,1)$ on $\left(16^{\prime}\right)$, then

$$
R E-A V=0
$$

and $\left(16^{\prime}\right)$ reduces to

$$
\frac{R}{A} x y-z=0 .
$$

As a result of (6), (10), (15) and (17) the expansions (1) become

$$
\begin{aligned}
& y=A x^{3}+\frac{A S}{R} x^{4}+\frac{A T}{R} x^{5}+\frac{A U}{R} x^{6}+\frac{A V}{R} x^{7}+F x^{8}+(9), \\
& z=R x^{4}+S x^{5}+T x^{6}+U x^{7}+V x^{8}+(9) .
\end{aligned}
$$

2. In $\$ 1$ we have, of course, not exhausted all the covariant figures determined by the neighborhood at $P$ of order up to and including eight. Before going on further we shall prove the following theorem.

THEOREM. If a proper unodal cubic surface has at $P$ a contact of order two with the quadric (16) and a contact of order eight with the curve (18), then the locus of the unode is the quadric (16).

Proof. Let $P_{0}\left(x_{0}, y_{0}, z_{0}\right)\left(y_{0} \neq 0\right)\left({ }^{3}\right)$ be any fixed point in space, then we may

$\left.{ }^{3}\right)$ It should be remarked here that $y_{0}=0$ possesses no geometrical significance since any plane, except $z=0$, through the singular tangent of (1) can be chosen as the coordinate plane $y=0$. If the section of the unodal cubic surface in consideration produced by any plane passing through the singular tangent is not composite, $P_{0}$ can not lie on the tangent except at $P$. Hereafter we take without loss of generality that $y_{0} \neq 0$. 
take, except for a projectivity, $y,\left(x y_{0}-x_{0} y\right),\left(y-y_{0}\right)$, and $\left(y z_{0}-y_{0} z\right)$ for coordinates of a point in space. If $P_{0}$ is the unode of a cubic surface, the equation of the latter is found to be

$$
\begin{aligned}
y & {\left[\alpha_{1}\left(x y_{0}-x_{0} y\right)+\alpha_{2}\left(y-y_{0}\right)+\alpha_{3}\left(y z_{0}-y_{0} z\right)\right]^{2} } \\
& +\beta_{1}\left(x y_{0}-x_{0} y\right)^{3}+\beta_{2}\left(x y_{0}-x_{0} y\right)^{2}\left(y-y_{0}\right)+\beta_{3}\left(x y_{0}-x_{0} y\right)^{2}\left(y z_{0}-y_{0} z\right) \\
(19) \quad & +\beta_{4}\left(x y_{0}-x_{0} y\right)\left(y-y_{0}\right)^{2}+\beta_{5}\left(x y_{0}-x_{0} y\right)\left(y-y_{0}\right)\left(y z_{0}-y_{0} z\right) \\
& +\beta_{6}\left(x y_{0}-x_{0} y\right)\left(y z_{0}-y_{0} z\right)^{2}+\beta_{7}\left(y z_{0}-y_{0} z\right)^{3}+\beta_{8}\left(y-y_{0}\right)\left(y z_{0}-y_{0} z\right)^{2} \\
& +\beta_{9}\left(y z_{0}-y_{0} z\right)\left(y-y_{0}\right)^{2}+\beta_{10}\left(y-y_{0}\right)^{3}=0,
\end{aligned}
$$

and the unodal plane is given by

$$
\alpha_{1}\left(x y_{0}-x_{0} y\right)+\alpha_{2}\left(y-y_{0}\right)+\alpha_{3}\left(y z_{0}-y_{0} z\right)=0,
$$

which may or may not pass through $P$. In the latter case we take, for brevity, $\alpha_{2}=1$.

When the cubic surface (19) has at $P$ a contact of order two with the quadric (16), substituting $R x y / A$ for $z$ in (19) leads to the following equations:

$$
\begin{gathered}
\beta_{10}=0, \quad \beta_{4}=0, \quad \beta_{2}=0, \\
-2\left(-\alpha_{1} x_{0}+\alpha_{3} z_{0}\right)+\beta_{5} x_{0} z_{0}-\beta_{8} z_{0}^{2}=0, \\
1+\beta_{9} z_{0}=0,
\end{gathered}
$$

and

$$
-2 \alpha_{1}-\beta_{5} z_{0}-\beta_{9} \frac{R y_{0}}{A}=0 .
$$

From (23) we know that $z_{0}$ cannot vanish and

$$
\beta_{9}=\frac{-1}{z_{0}} .
$$

Consequently, (22) and (23) give rise to

$$
\beta_{\mathrm{b}}=\frac{1}{z_{0}}\left(\frac{R}{A} \frac{y_{0}}{z_{0}}-2 \alpha_{1}\right)
$$

and

$$
\beta_{8}=\frac{1}{z_{0}^{2}}\left(-2 \alpha_{3} z_{0}+\frac{R x_{0} y_{0}}{A z_{0}}\right) .
$$

After substituting (18) into (19) it follows that the coefficient of the term $x^{r}$ depends only upon the terms $x^{\alpha} y^{\beta} z^{\gamma}$ in (19) where

$$
\alpha+\beta+\gamma \leqq 3, \quad \alpha+3 \beta+4 \gamma=r \quad(\alpha, \beta, \gamma \geqq 0) .
$$


In case $r=3$, there are, in fact, two possibilities: firstly, $\beta=1, \alpha=0, \gamma=0$ and secondly, $\beta=0, \alpha=3, \gamma=0$. Because the coefficient of the term $y$ in (19) has already been reduced to zero by (23), we obtain, when the order of contact between (18) and (19) is three, that

$$
\beta_{1}=0 \text {. }
$$

Now the equation (19) of the unodal cubic surface takes the form

$$
\begin{aligned}
& y\left[\alpha_{1}\left(x y_{0}-x_{0} y\right)+\left(y-y_{0}\right)+\alpha_{3}\left(y z_{0}-y_{0} z\right)\right]^{2} \\
& \quad+\beta_{3}\left(x y_{0}-x_{0} y\right)^{2}\left(y z_{0}-y_{0} z\right) \\
& \quad+\frac{1}{z_{0}}\left(\frac{R y_{0}}{A z_{0}}-2 \alpha_{1}\right)\left(x y_{0}-x_{0} y\right)\left(y-y_{0}\right)\left(y z_{0}-y_{0} z\right) \\
& \quad+\beta_{6}\left(x y_{0}-x_{0} y\right)\left(y z_{0}-y_{0} z\right)^{2}+\beta_{7}\left(y z_{0}-y_{0} z\right)^{3}-\frac{1}{z_{0}}\left(y z_{0}-y_{0} z\right)\left(y-y_{0}\right)^{2} \\
& \quad+\frac{1}{z_{0}}\left(-2 \alpha_{3} z_{0}+\frac{R x_{0} y_{0}}{A z_{0}}\right)\left(y z_{0}-y_{0} z\right)^{2}\left(y-y_{0}\right)=0,
\end{aligned}
$$

which actually contains only the terms $x^{\alpha} y^{\beta} z^{\gamma}$ where $\alpha, \beta$, and $\gamma$ satisfy

$$
\alpha+3 \beta+4 \gamma>3 \text {. }
$$

Consequently the necessary and sufficient condition that (30) and (18) should have at $P$ a contact of order four depends upon the coefficients of the terms $x y$ and $z$ in (30). A simple calculation shows that, except for

$$
\left(-2 y_{0}^{2} \alpha_{1}-\beta_{5} y_{0}^{2} z_{0}\right)\left(x y-\frac{R}{A} z\right) \text {, }
$$

(30) does not contain $x y$ and $z$. Because $x y-R z / A=0$ and (18) have at $P$ a contact of order eight, (30) and (18) should naturally have át $P$ a contact of order four. In order that the order of contact between (30) and (18) be elevated to five, the necessary and sufficient condition will contain the coefficients of the terms $x^{2} y$ and $x z$ and is easily found to be

$$
\alpha_{1}^{2}+\beta_{3} z_{0}+\frac{y_{0}}{z_{0}} \cdot \frac{R}{A}\left(\frac{R y_{0}}{A z_{0}}-2 \alpha_{1}\right)=0 .
$$

Hence the terms $x^{2} y$ and $x z$ contained in (30) become

$$
\left(x^{2} y-\frac{A}{R} x z\right)\left(\alpha_{1}^{2} y_{0}^{2}+\beta_{3} y_{0}^{2} z_{0}\right)
$$

which is an infinitesimal of order 10 when (18) is substituted in it.

When (18) and (30) have at $P$ a contact of order six, besides (32) it requires a further condition 


$$
\beta_{3}=0 \text {. }
$$

Combining (32), (34), and (25) gives

$$
\alpha_{1}=R y_{0} / A z_{0}
$$

and

$$
\beta_{5}=-R y_{0} / A z_{0}^{2} .
$$

Under (32) and (34) the left side of (30) becomes an infinitesimal in $x$ of order seven when we take account of (18). To pay attention to the integral solution of

$$
\alpha+\beta+\gamma \leqq 3, \quad \alpha+3 \beta+4 \gamma=7 \quad(\alpha, \beta, \gamma \geqq 0),
$$

we find that whether (18) and (30) can have a contact of order seven is determined by a linear relation of the coefficients of the terms $y z$ and $x y^{2}$ in (30). In other words, when the order of contact between (18) and (30) is seven, $\beta_{6}$ should vanish and (30) does not contain $x y^{2}$ or $y z$ except for

$$
\left(x y^{2}-\frac{A}{R} y z\right)\left[\frac{R y_{0}^{2}}{A z_{0}}\left(-\frac{R x_{0} y_{0}}{A z_{0}}+2 t_{0}+2 \alpha_{3} z_{0}\right)\right] .
$$

Similarly the ratio of the coefficients of the terms. $z^{2}$ and $x y z$ in (30) must be equal to $R / A$ if (30) and (18) have at $P$ a contact of order eight, that is

$$
-2 \alpha_{1} \alpha_{3}+\frac{R y_{0}}{A z_{0}^{2}}-\frac{R}{A} \frac{y_{0}}{z_{0}^{2}}\left(-2 \alpha_{3} z_{0}+\frac{R x_{0} y_{0}}{A z_{0}}\right)=0 .
$$

which is equivalent to $R x_{0} y_{0}-A z_{0}=0$.

If the plane (20) passes through $P$, the unodal cubic surface in consideration must decompose into two coincident planes $y z_{0}-y_{0} z=0$ and another plane $p z+q y=0$. Hence the theorem is proved.

3. In order to investigate other covariant properties determined by the neighborhood at $P$ of order no more than eight we select now, contrary. to the last section, such unodal cubic surfaces to approximate $\Gamma$ at $P$ as are even not tangent to $z=0$ at $P$. Let $\left(x_{0}, y_{0}, 0\right)$ be the unode; a unodal cubic surface of this character may. be given by the equation

$$
\begin{aligned}
& y\left[\alpha_{1}\left(x y_{0}-x_{0} y\right)+\alpha_{2}\left(y-y_{0}\right)+\alpha_{3} y_{0} z\right]^{2}+\beta_{1}\left(x y_{0}-x_{0} y\right)^{3} \\
& \quad+\beta_{3} z\left(x y_{0}-x_{0} y\right)^{2}+\beta_{5} z\left(y-y_{0}\right)\left(x y_{0}-x_{0} y\right)+\beta_{6} z^{2}\left(x y_{0}-x_{0} y\right) \\
& \quad+\beta_{7} z^{3}+\beta_{8} z^{2}\left(y-y_{0}\right)+\beta_{9} z\left(y-y_{0}\right)^{2}=0,
\end{aligned}
$$

provided it has at $P$ a contact of order two with the curve (18).

If $z=0$ be different from the tangent plane of (39) at $P, \alpha_{2}$ will be nonvanishing, so that we put, for brevity, 


$$
\alpha_{2}=1 \text {. }
$$

Imposing the condition that (39) and (18) should have at $P$ a contact of order three we have

$$
\beta_{1}=-A / y_{0}
$$

where $y_{0} \neq 0$.

The unodal plane of (39) intersects $y=0, z=0$ at $\left(1 / \alpha_{1}, 0,0\right)$ which corresponds under $P_{5}$ to

$$
R y-A \alpha_{1} z=0 .
$$

In this section we take this plane for the tangent plane of (39) at $P$, then

$$
\beta_{0}=-\frac{A \alpha_{1}}{R} .
$$

Since the necessary and sufficient condition that (39) and (18) have a contact of order four is

$$
\alpha_{1}=S / 3 R \text {, }
$$

we obtain the covariant point $(S / 3 R, 0,0)$, which coincides with $Z_{5}$ defined previously $\left({ }^{4}\right)$. Hence we have proved the following theorem.

THEOREM. The point of intersection $N$ of the singular tangent $y=0, z=0$ and the unodal plane of the cubic surface (39) is independent of the selection of the unode on the plane $z=0$ if the surface (39) has at $P$ a contact of order eight with (18) and at $N$ a contact of order one with (16). The point $N$ must coincide with $Z_{5}$ unless $N \equiv P$.

This is an analogous theorem to that regarding the covariant point $O_{4}\left({ }^{5}\right)$ in case of plane curves.

Taking (42) for $y=0, \alpha_{1}, S$ and $\beta_{9}$ reduce to zero and (39) becomes

$$
\begin{aligned}
& y\left[\left(y-y_{0}\right)+\alpha_{3} y_{0} z\right]^{2}+\frac{A}{y_{0}}\left(x_{0} y-y_{0} x\right)^{3} \\
& \quad+\beta_{3} z\left(x y_{0}-x_{0} y\right)^{2}+\beta_{5} z\left(x y_{0}-x_{0} y\right)\left(y-y_{0}\right)+\beta_{6} z^{2}\left(x y_{0}-x_{0} y\right) . \\
& \quad+\beta_{7} z^{3}+\beta_{8} z^{2}\left(y-y_{0}\right)=0 .
\end{aligned}
$$

If (45) has further at $P$ a contact of order five with (18), we obtain

$$
\frac{A T}{R} y_{0}+3 A^{2} x_{0}-\beta_{5} R y_{0}^{2}=0 .
$$

(4) See Chang, loc. cit.

(5) E. Bompiani, Per lo studio proiettivo-differenzialle delle singolarita, Bollettino dell'UnioneMatematica Italia vol. 5 (1926) pp. 118-120. 
The polar quadric of the covariant point $Z_{5}(1,0,0)$ with respect to (45) degenerates when and only when $\beta_{5}=0$. From (46) follows the covariant line

$$
\frac{T}{R} y+3 A x=0, \quad z=0,
$$

where $T \neq 0$, if (47) does not coincide with (9).

Under $P_{7}$ the point of intersection of the line $z=0, x=0$ and the line $Z_{5} P_{0}$ corresponds to the plane

$$
R y_{0} x-A t_{0} z=0
$$

when $y=0,(48)$ and the tangent plane of $(45)$ at $Z_{5}$ belong to a pencil, we have

$$
\beta_{3}=-3 A^{2} t_{0} / R y_{0} ;
$$

but the necessary and sufficient condition that (45) and (18) have at $P$ a contact of order six gives that

$$
\beta_{8}=\frac{1}{R y_{0}}\left(2 t_{0} A^{2}-\frac{A}{R} U y_{0}\right) .
$$

Combining (49) and (50) we obtain a covariant line

$$
z=0, \quad y \frac{A V}{R}+\frac{A T}{R^{2}}(-T y+A R x)=0 .
$$

Taking this for $t=0, z=0$ we have

$$
U=0 .
$$

With respect to the local coordinate tetrahedron so far geometrically determined, the semi-canonical expansions of the curve may be written as

$$
\begin{aligned}
& y=A x^{3}+\frac{A T}{R} x^{5}+\frac{A V}{R} x^{7}+B^{\prime} x^{8}+(9), \\
& z=R x^{4}+T x^{6}+V x^{8}+W x^{9}+(10) .
\end{aligned}
$$

The locus of the node of (45) is easily found to be

$$
\begin{gathered}
z=0, \\
\frac{A V}{R} y+(-T y+A R x) \frac{A T}{R^{2}}=0,
\end{gathered}
$$

if (45) has at $P$ a contact of order seven with (53) and the unodal plane of (45) passes through $(1,0,0)$ and $(0,0,1)$.

Among the unodal cubic surfaces (45), a special class may be formed such that $x=0, y=0$ is an asymptotic tangent. A cubic surface of this class has at $P$ a contact of order eight when and only when the node lies on the conic 


$$
B^{\prime} y^{2}+3 A^{3} y t-A^{3} x t=0, \quad z=0 .
$$

Suppose that $(1,1,0)$ lies on $(47)$ and $(55)$, and $(1,1,1)$ on $(16)$, then

$$
B^{\prime}=-2 A^{8}, \quad A=R, \quad T=-3 A^{3} .
$$

In consequence we obtain the canonical expansions of the curve

$$
\begin{aligned}
& y=A x^{8}-3 A^{2} x^{5}+V x^{7}-2 A^{3} x^{8}+(9), \\
& z=A x^{4}-3 A^{2} x^{6}+V x^{8}+(9) .
\end{aligned}
$$

4. Let $(\tau, \xi, \eta, \zeta)$ be homogeneous coordinates of a point in space defined by putting

$$
\xi / \tau=x, \quad \eta / \tau=y, \quad \zeta / \tau=z .
$$

In the pencil $\lambda \xi+\eta=0$, the planes $\xi=0, \eta=0$ and $-\xi+\eta=0$ have their particular geometrical significances. When $\bar{\lambda}=-A x^{3}+(4)$, the plane $\lambda \xi+\eta=0$ passes through the point on (18) infinitely near $P$. The double ratio of these four planes will be denoted by $D_{1}$. Similarly we denote the double ratio of $\xi=0, \zeta=0,-\xi+\zeta=0$ and

$$
\zeta-\left(A x^{4}+(5)\right) \xi=0
$$

by $D_{2}$. It follows at once that, save for an infinitesimal,

$$
A=D_{1}^{4} / D_{2}^{8} \text {. }
$$

Furthermore, in the plane $\zeta=0$ the double ratio $D_{3}$ of the four lines $\xi=0$, $\eta=0, \xi=\eta$ and (54) furnishes a geometrical interpretation of the invariant $V / A^{3}$ :

$$
3+D_{8}=\frac{V}{3 A^{3}} .
$$

National University of Chekiang,

Meitan, Kweichow, China. 